\title{
DIFFERENT CHIP INTERCONNECTING TECHNIQUES
}

\author{
HANS DANIELSSON \\ Saab-Scania AB, Aerospace Division, 58188 Linköping, Sweden
}

(Received February 28, 1980)

\begin{abstract}
It is shown that in order to interconnect more than 25 I.C. chips (SSI and MSI) or to use LSI chips together with other kind of chips, the chips must be tested before mounting. Then only TAB- or CC-techniques can be used. The advantages and disadvantages of the different techniques are discussed.
\end{abstract}

\section{INTRODUCTION}

The choice of chip interconnecting technique in manufacturing hybrid circuits used on an electronic system has strong influence on the systems' parameters. Among such parameters are

System cost (total hardware cost)

System weight and volume

System reliability

It is important to look upon the interconnecting technique not only from a circuit point of view but also from a system point of view. Firstly, the reduction of printed wiring boards, back panels and mechanics etc. which is obtained by increased packaging density can be put into its right economic and technical perspective. Consider for example the total hardware manufacturing cost for a digital system built using hybrid techniques. If this cost is

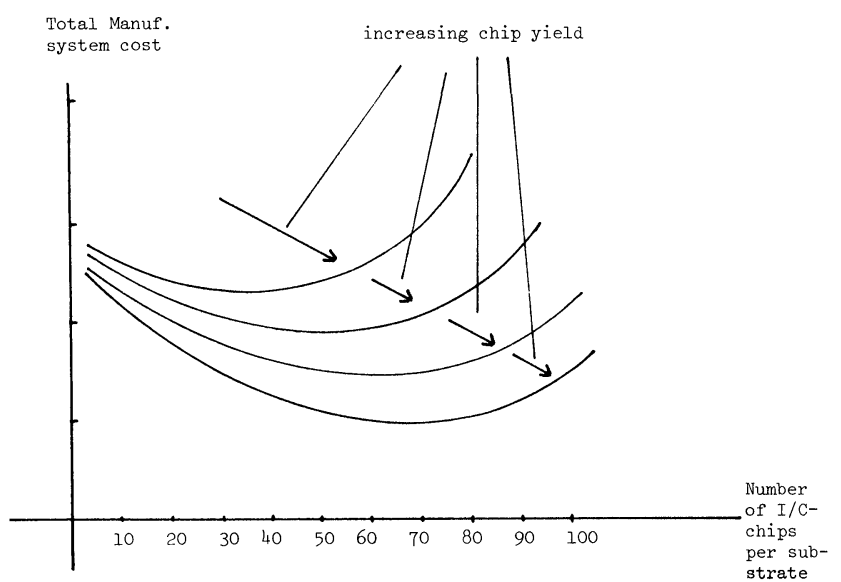

FIGURE 1 Importance of chip yield in hybrid circuit manufacturing. plotted as a function of the number of I.C. chips per hybrid substrate, Figure 1 is obtained.

To look in'more detail at the chip yield, consider a substrate with N I.C chips.

There are $n_{1}$ chips of type $1, n_{2}$ chips of type 2 etc. Let $y_{1}$ be the probability that each chip of type 1 will be electrically good after bonding in the whole temperature range, $y_{2}$ the probability of chip type 2 and so on. Then, the probability that the substrate containing $N$ chips will be electrically good is

$$
\begin{aligned}
& y_{T F}=\left(y_{1}\right)^{n_{1}} \cdots\left(y_{n}\right)^{n} \cdot y_{s} \quad \text { (1) } y_{s}=\begin{array}{c}
\text { substrate } \\
\text { yield }
\end{array} \\
& N=n_{1}+n_{2}+\ldots n_{n}
\end{aligned}
$$

Let for simplicity $y_{1}=y_{2}=\cdots y_{n}=y_{I / C}$

Then Eq. (1) reduces to

$$
y_{T F}=\left(y_{I / C}\right)^{N}
$$

Using Eq. (3) Table I is obtained.

It is the experience of Saab-Scania using wire bonded digital chips of SSI- and MSI-complexity that $y_{I / C} \sim 0.975$. This is a mean yield figure taken over several production lots. Furthermore that yield is obtained after visual inspection to Mil-Std2010 and after electrical test on sample basis.

If for example there are 25 chips of SSI or MSI complexity on a substrate and if $y_{I / C} \sim 0.975$ Table I shows that only $53 \%$ of the assembled hybrids do not need rework. But imagine what will happen with a production lot when the yield for some chips is $\sim 0.90$.

This example shows that using wire bonding of digital chips (no 100\% electrical test of the chip before bonding) of SSI and MSI complexity a practical limit of the complexity for the hybrid circuit is less than 25 to 30 I.C. chips. 
TABLE I

Yield of $N$ chip substrates.

\begin{tabular}{|c|c|c|c|c|c|c|c|c|}
\hline \multirow{2}{*}{\multicolumn{2}{|c|}{$\begin{array}{l}\text { Chip yield } \\
y_{I / C}\end{array}$}} & \multicolumn{2}{|c|}{$y_{T F}=\left(y_{I / C}\right)^{N}$} & \multicolumn{5}{|c|}{$y_{s}=1.0$} \\
\hline & & $N=5$ & $N=15$ & $N=20$ & $N=25$ & $N=40$ & $N=60$ & $N=100$ \\
\hline \multicolumn{2}{|l|}{0.999} & 0.995 & 0.985 & 0.980 & 0.97 & 0.96 & 0.94 & 0.90 \\
\hline \multicolumn{2}{|l|}{0.99} & 0.95 & 0.86 & 0.82 & 0.78 & 0.67 & 0.55 & 0.37 \\
\hline \multicolumn{2}{|c|}{$0.975^{*}$} & 0.88 & 0.68 & 0.60 & 0.53 & 0.36 & 0.22 & 0.08 \\
\hline \multicolumn{2}{|l|}{$0.95 *$} & 0.77 & 0.46 & 0.36 & 0.28 & 0.13 & 0.05 & \\
\hline \multicolumn{2}{|l|}{0.90} & 0.59 & 0.20 & 0.12 & 0.07 & 0.01 & & \\
\hline \multicolumn{2}{|l|}{0.85} & 0.44 & 0.09 & 0.04 & 0.02 & 0.001 & & \\
\hline \multicolumn{2}{|l|}{0.80} & 0.33 & 0.035 & 0.01 & & & & \\
\hline 0.75 & $\approx$ & 0.24 & 0.013 & & & & & \\
\hline 0.70 & 灵 & 0.17 & 0.004 & & & & & \\
\hline 0.60 & $\stackrel{1}{1}$ & 0.08 & & & & & & \\
\hline 0.50 & \& & 0.03 & & & & & & \\
\hline
\end{tabular}

*SSI and MSI

For linear chips the yield can be much lower than 0.95 depending on used electrical parameters.

If the hybrid technology is to be useful for future electronic systems, it is important that LSI-chips can be incorporated in the hybrid circuit. Suppose that a hybrid circuit with 8 SSI and MSI chips is to be interconnected with a 2 LSI chip. If the chip yield is 0.975 and 0.5 respectively Eq. (1) gives the yield for this circuit.

$$
y_{T F}=(0.975)^{8} \cdot(0.5)^{2}=0.21
$$

Therefore this circuit is too complex to produce in quantities.

This discussion shows that there has to be a method of testing the chips electrically before mounting them on a substrate in the following cases:

- More than 25 I.C. chips/substrate (SSI or MSI)

- More than 6 linear chips/substrate

- LSI-chips are used in combination with other kinds of chips.

Note that only the TAB-technique (Tape Automated Bonding) or the Chip Carrier technique permits testing of chips before mounting on a substrate.

\section{WIRE BONDING}

Wire bonding is a very flexible method. It is easy to use chips from different manufacturers in spite of different chip layouts. Automated wire bonding techniques are increasingly used and are cost effective when the number of chips is not too high. ${ }^{1,2}$ However, the wire bonding step is one of

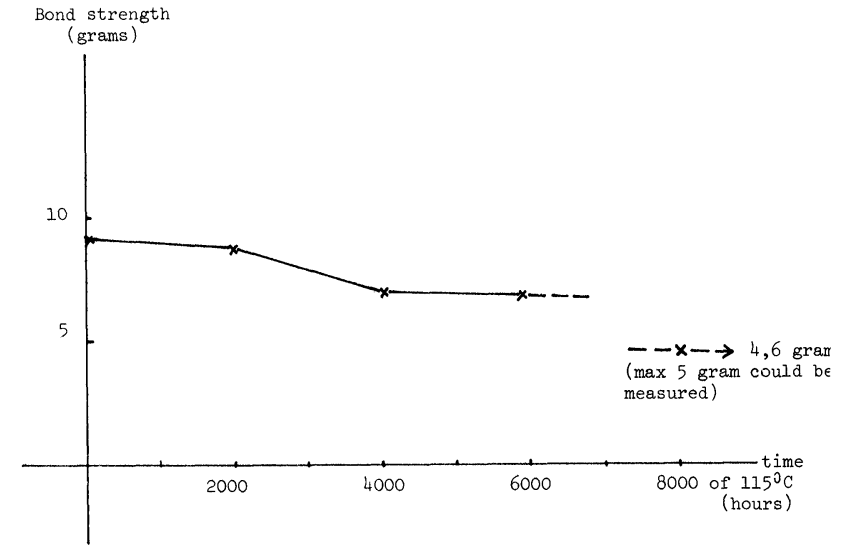

FIGURE 2 Mean bond strength as function of time at $115^{\circ} \mathrm{C}$.

the most unreliable steps in the hybrid circuit manufacturing process. Koudounaris ${ }^{3}$ reports that $23 \%$ of all field failures for hybrid circuits are wire bonding related failures.

Figure 2 shows the bond strength as a function of time at $115^{\circ} \mathrm{C}$. In this case 1 mil gold wires are thermosonically bonded to Al-metallization on I.C. chips.

As can be seen from Figure 2 the mean bond strength seems to be very satisfactory. However, the bond strength distribution of 327 bonds after $8000 \mathrm{~h}$ at $115^{\circ} \mathrm{C}$ is shown in Figure 3.

As can be seen from Figure 3 some of the bonds have much lower strength than the others. Analyses show that low strength bonds often are found on chips where approximately 13 bonds have bond 


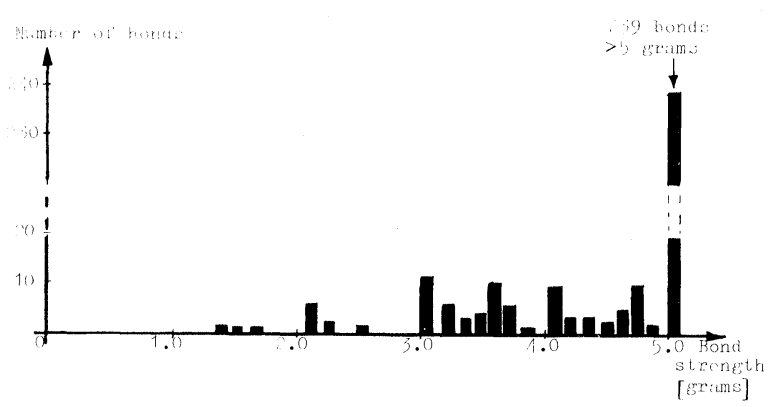

FIGURE 3 Bond strength distribution for 327 bonds after $8000 \mathrm{~h}$ at $115^{\circ} \mathrm{C}$.

strengths $>4.5$ gram and 1 bond $<2$ gram. In some cases we have been able to show that the bonding pad with low bond strength has had a thin layer of passivation glass only at this low strength pad. When probing such a chip the probe has gone through the thin glass layer and made an electrical contact. When bonding on this pad the bonding has taken place only in the probe mark. Another reason for low bond strength on some pads can be photo resist residues.

Unfortunately wire bonds always degrade with time and temperature and there is no method to screen out low strength bonds. Centrifuging hybrid circuits has very little effect on wire bonds as a hybrid circuit seldom can withstand the $>30000 \mathrm{~g}$ needed to apply a screening force on the bonds.

\section{TAPE AUTOMATED BONDING (TAB)}

The TAB-bonding technique is used to a great extent in the semi-conductor industry. Today several million TAB-chips per week are bonded into plastic DIL-packages. The hybrid circuit manufacturers have also been using TAB-chips for some years. ${ }^{4,5,6}$ Figure 4 shows a complex linear circuit using TAB-bonded chips. Figure 5 shows an arithmetic unit for a microcomputer using TAB-chips. Note that in this circuit some of the chips are wire bonded. The circuit is manufactured at Saab-Scania.

The TAB-technique is inherently more reliable than wire bonding ${ }^{5,6}$ partly due to the mechanically rigid structure of the TAB-fingers. However, different semiconductor manufacturers use different bump materials and different bump height etc. This can cause problems for the hybrid manufacturer using TAB-chips from different vendors.

Another problem is the lack of standardizations of the OLB-pattern (Other Lead Bonding) for TAB-chips bonded to hybrid substrates.

This implies that TAB-bonding is a good technique to use for a hybrid manufacturer who uses a limited number of TAB-chip types and uses them in a large quantity each year. However, it is not easy to use the TAB-technique "on the system level" where all the I.C. chips used in a project have to be in TAB-form. In this case the hybrid manufacturer has to get TAB-chips of many types from several semiconductors. This will cause problems of availability of TAB-chips

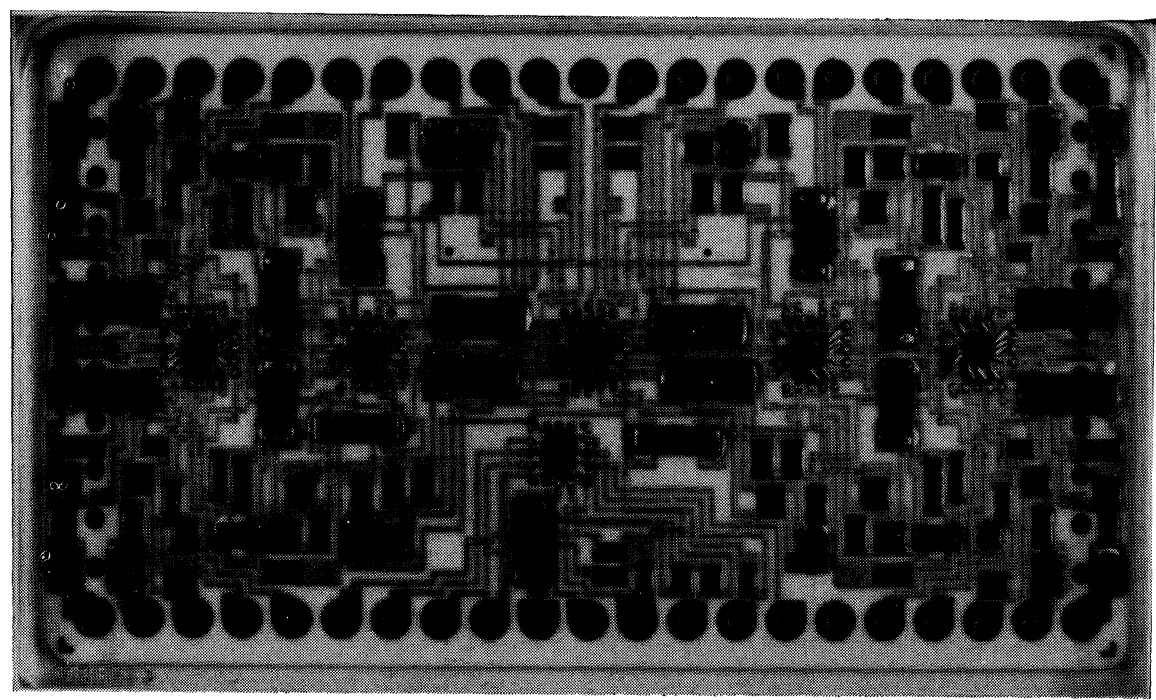

FIGURE 4 Linear circuit using 6 TAB-chips. (Saab-Scania) 


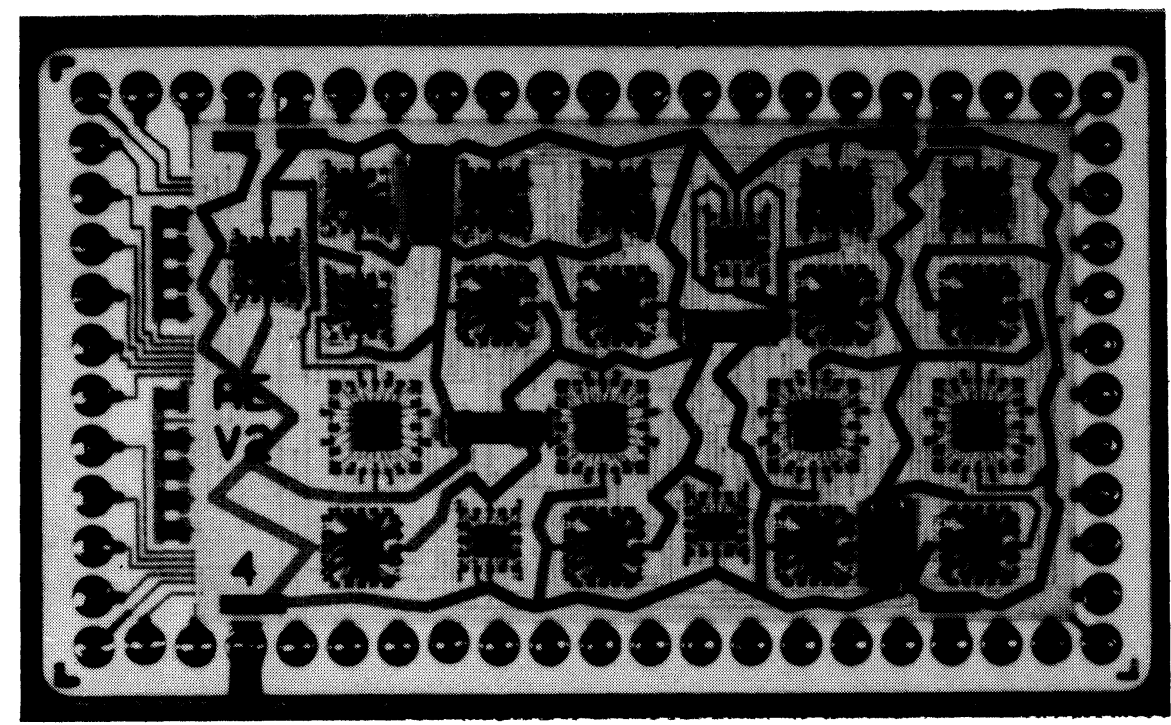

FIGURE 5 Arithmetic unit for a microprocessor using TAB-chips. (Saab-Scania)

and also a compatibility problem. For example, the manufacturer using 4 in wafers has different chip thickness from a manufacturer using 3 in wafers which makes changes in the length of the TAB-fingers necessary. However, there is no doubt that the TAB-technique will be increasingly used in hybrid circuit manufacturing in the future.

\section{CHIP CARRIER PACKAGE (CC-PACKAGE)}

The use of chip carriers (also called ceramic chip carrier) is a technique which has gained much interest from the aerospace and high-reliability electronic industry. The semiconductor industry also considers the CC-technique as essential for many reasons. One reason is that the CC-package is the only practical package for LSI circuits with more than 64 leads. Other reasons are the much lower cost for a CC-package compared to the corresponding DIL-package, however the main reason is the high degree of automatization which the CC-packages permit in the semiconductor plant compared to the DIL-packages. Some of the big semiconductor industries are now working intensively on automating the production of CC-packages. Therefore the cost of a CC-package is said to be at least $1 \$$ less than the corresponding chip in a DIL-package. The CC-package will become a standard package supplied by the semiconductor industry during 1978-1980.
The CC-package offers several advantages to the hybrid circuit manufacturer. One important advantage, especially for LSI-circuits, is the possibility of testing the CC-package electrically before mounting the circuits on a substrate.

Burn-in can easily be done on CC-packages as well as on TAB-chips. However, the chips are much better protected, mechanically and chemically, during and after burn-in if the chips are mounted in hermetic sealed CC-packages. This is an essential advantage of the CC-technique over the TAB-technique seen from a reliability point of view.

At first sight the packaging density with CC-packages seems to be much lower than hybrids with wire bonded or TAB-chips. However, CC-packages can be mounted on both sides of the substrate. ${ }^{7}$ Therefore the CC-technique can give higher volume packaging densities than other techniques.

The solder joints between the CC-packages and the substrate are easy to visually inspect to assure a good joint. The positioning of the CC-package on the base substrate is not critical because errors in both translation and rotation are corrected by the surface tension of the molten solder during the reflow process.

A great advantage of the CC-technique is the simplicity of replacing a faulty CC-package even at the field repair level. This can be done either by a hot-air gun, a hot plate or a specially formed soldering iron. ${ }^{7,8}$ An important property of the 


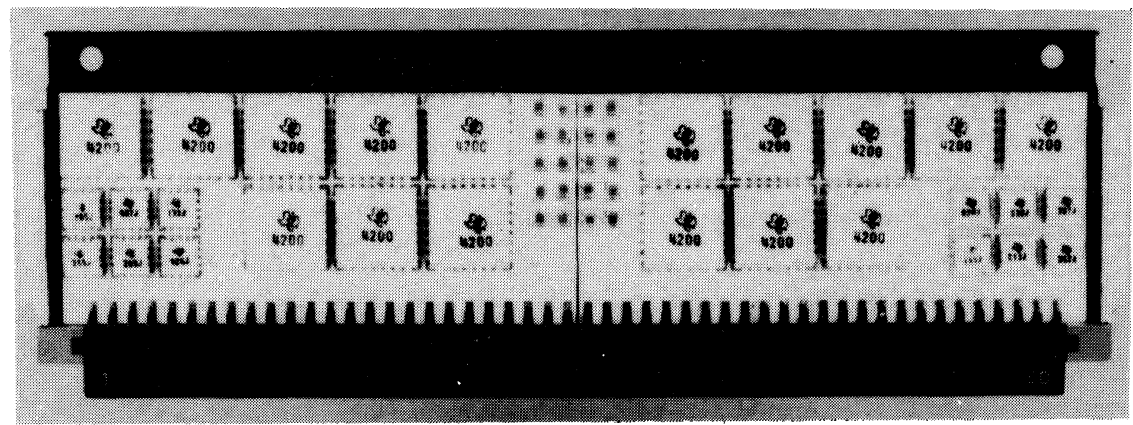

FIGURE 6 Chip Carriers in NAFI module. Courtesy Texas Instruments.

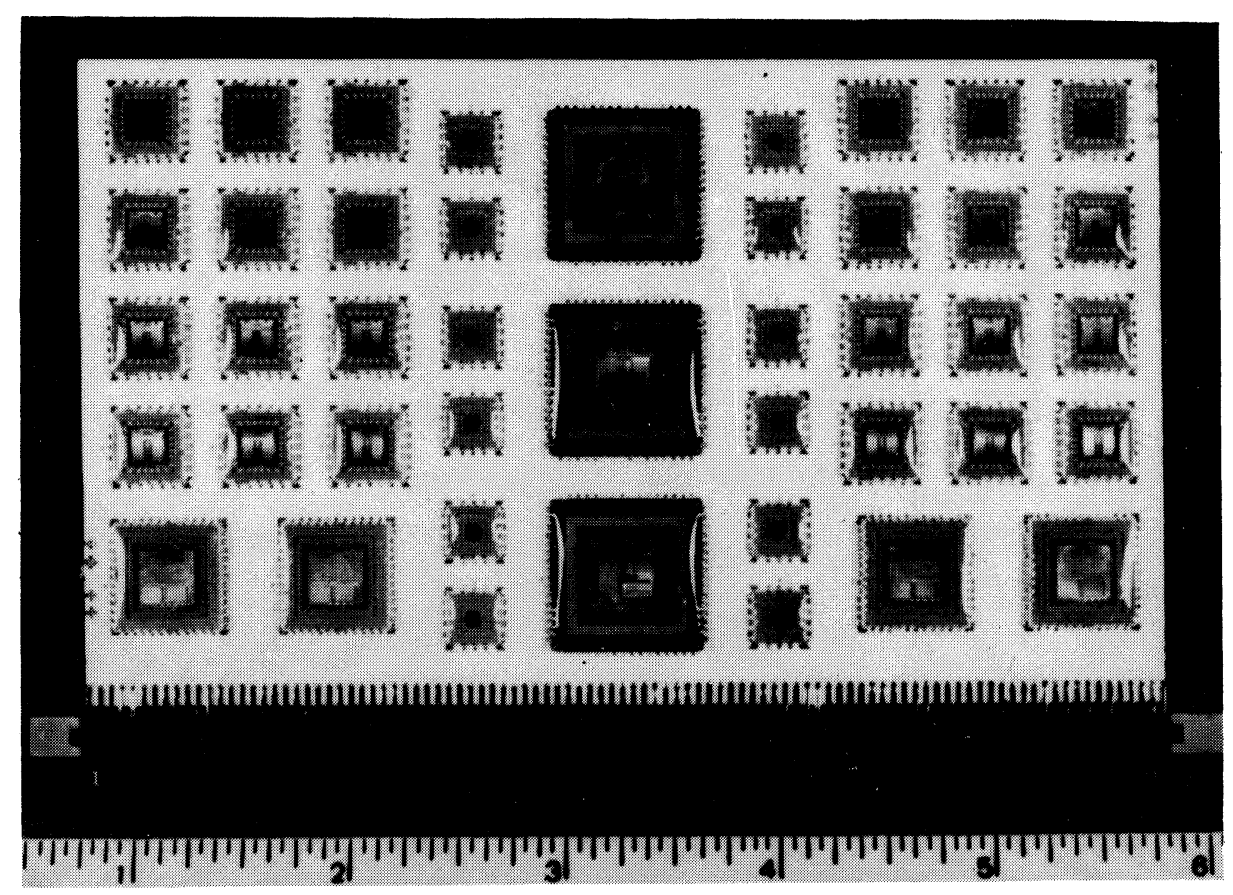

FIGURE 7 CC-packages on 5 in $\times 4$ in substrate. No lid on the packages for demonstration purpose. Courtesy RCA.

CC-technique is that in prototype or preproduction manufacturing it is possible to cut conductors on the substrate and solder small wire jumpers between CC-packages.

CC-packages are currently available from at least 3 manufacturers. There is a JEDEC standard on the physical size for the CC-packages with leads between 16 and 156 .

The hybrid circuit manufacturer can then buy naked chips (all types are in principle available) together with standard CC-packages. Then he can mount and bond the chips in the CC-packages and then seal the packages. All steps in manufacturing are using standard hybrid techniques. This can be done until the hybrid circuit manufacturer can buy the CC-packages directly from the semiconductor manufacturer. For examples of big substrates using CC-packages, see Figures 6 and 7. 


\section{CONCLUSIONS}

It is important to look upon different chip interconnecting techniques not only from a circuit but also from a total system point of view. This approach often implies that more than 25 MSI and SSI chips should be mounted on each substrate. Another important case from the system point of view is to incorporate LSI chips together with other kinds of chips. Both cases require that all chips are electrically tested before mounting them on the substrate. The only chip interconnecting techniques which permit this are

\section{1) $T A B$-technique}

2) CC-technique

The choice between TAB- and CC-techniques depends on several system parameters such as number of chip types, number of produced systems and reliability demand. The CC-technique should be chosen for high reliability applications whereas the TAB-technique is perhaps the best for medium reliability and high volume production application. For small serial application the CC-technique is a good choice.

Many semiconductor houses are now tooling up for highly automated production of CC-packages. Therefore the CC-package will become a standard item supplied by the semiconductor houses during 1979-80.

The CC-packages offer many advantages from the total system point of view compared with DIL packages. Hence the CC-packages are of great importance to the hybrid circuit industry. With the CC-packages the hybrid circuit technique really can grow and become the packaging medium for the LSI and VLSI generations.

\section{ACKNOWLEDGEMENTS}

The author is very grateful to Mr Jon Prokop TI and Mr John Bauer RCA for very helpful discussions.

\section{REFERENCES}

1. F. W. Beyerlein, "New developments in automatic wire bonding equipment", Electron. Packag. and Prod., January 1978 , pp. 54-67.

2. S. Khadpe, "Yield and throughput considerations for automated wire bonding of multi chip hybrids", ISHM Proc. Sept. 1978, pp. 157-160.

3. A. Koudounaris, et al, "Pretesting of active device chips for use in hybrids", ISHM Proc. Sept. 1978, pp. 10-17.

4. G. DeHaine and K. Kurzweil, "Tape automated bonding moving into production", ISHM Proc. 1975.

5. C. Bernadotte, "Development of the TAB-process at Saab-Scania", 28th Electron. Comp. Conf., April 24-26, 1978, pp. 151-158.

6. J. M. Montante, et al, "Storage and life tests of thermocompression tape automated bonded leads to various gold metallizations", ISHM Proc. 1978, pp. 169-175.

7. John A. Bauer, "Leadless carrier applications for avionics". Presented at AIAA digital avionics conference, Los Angeles Nov. 2-4, 1977.

8. Jon S. Prokop and Dale W. Williams "Chip carriers as a means for high density packaging”, ISHM Proc. 1977, pp. 262-269. 

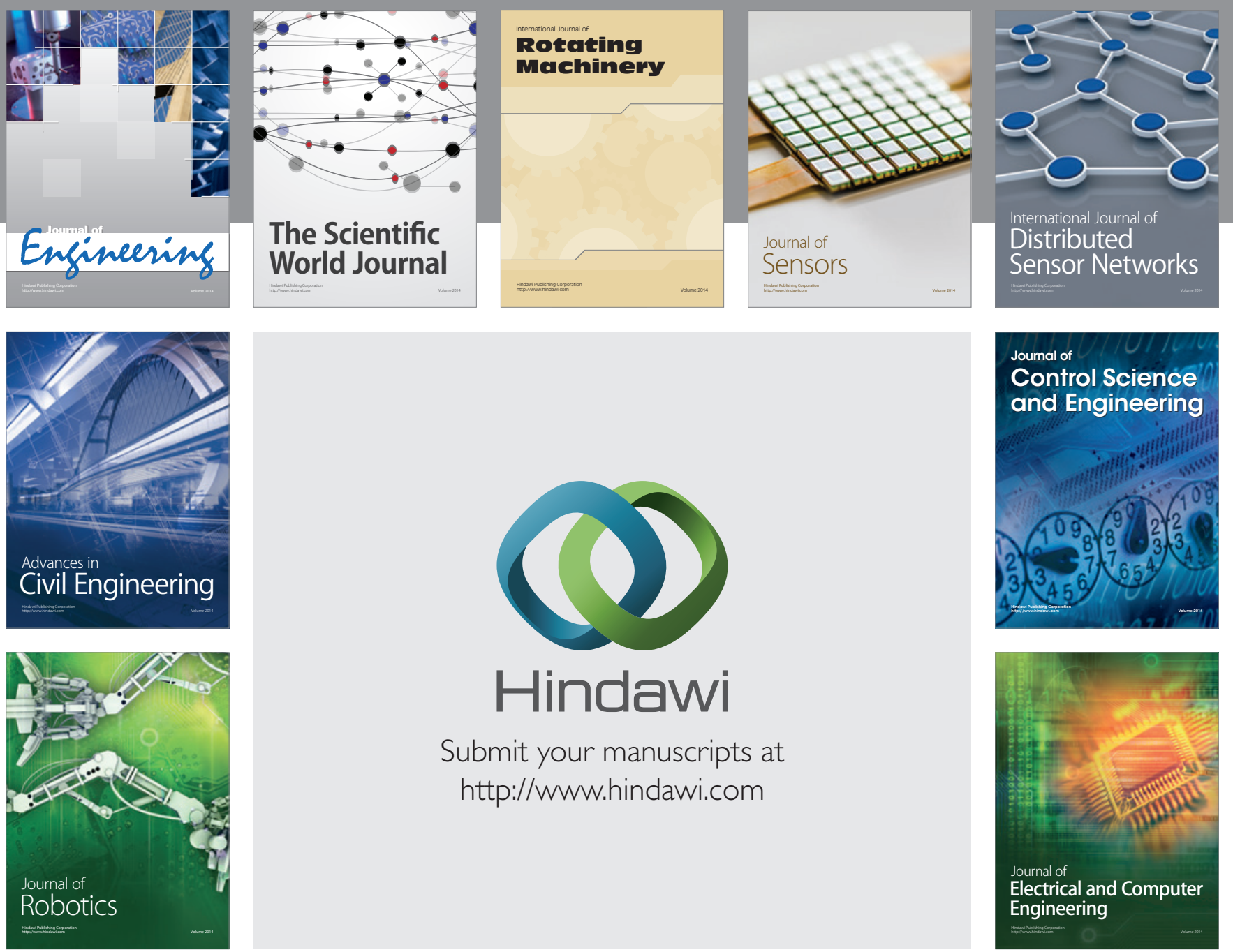

Submit your manuscripts at

http://www.hindawi.com
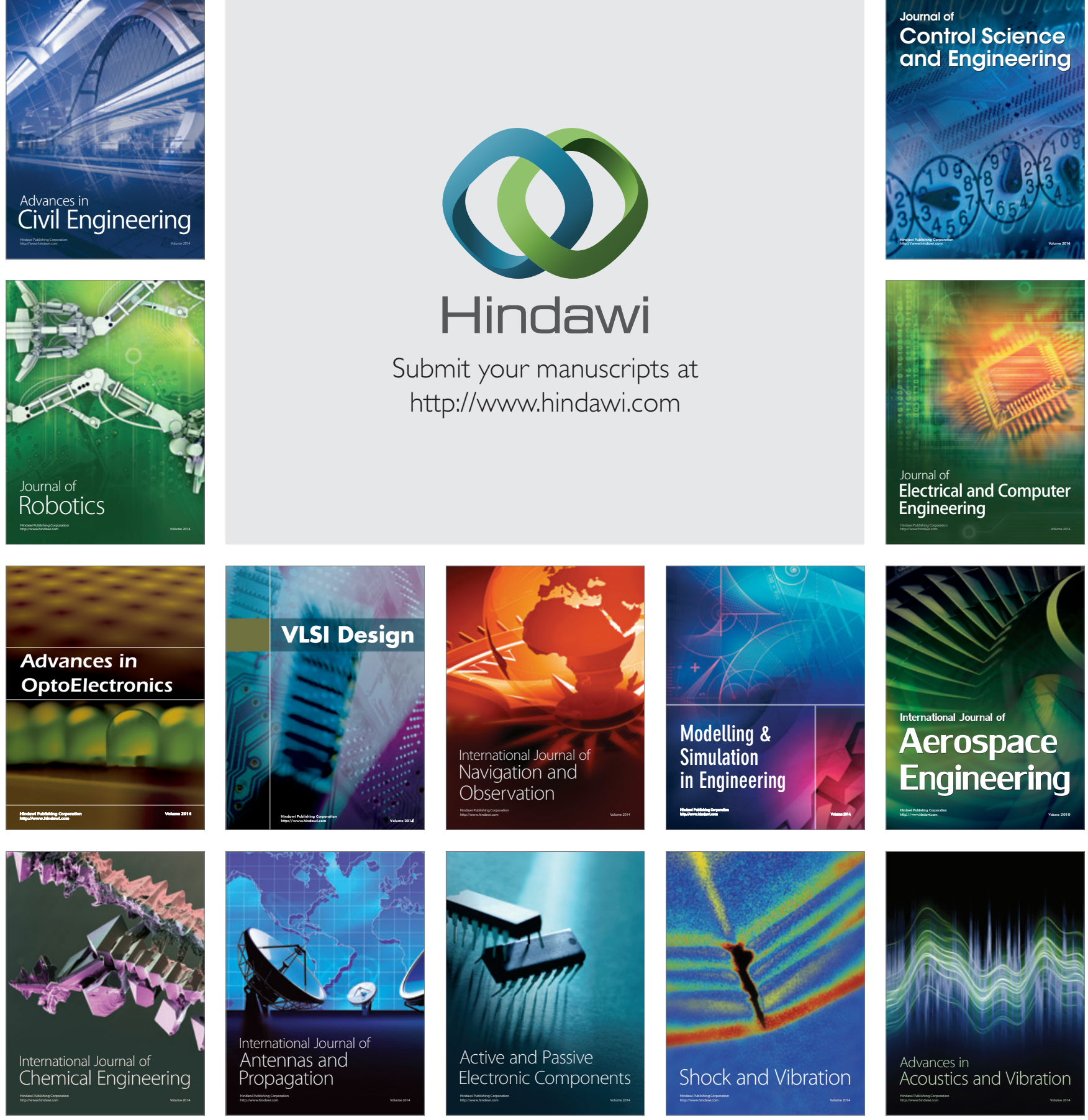